\title{
Padrões alimentares e imagem corporal em mulheres frequentadoras de academia de atividade física
}

\author{
Marcia Cristina Dominguez Fernandes Pinto de Souza - Faculdades Integradas Fafibe, Bebedouro/SP, Brasil \\ Laura Vilela e Souz̨a - Universidade Federal do Triângulo Mineiro, Uberaba/MG - Brasil \\ Sabrina Martins Barroso - Universidade Federal do Triangulo Mineiro, Uberaba/MG - Brasil \\ Fabio Scorsolini-Comin - Universidade Federal do Triângulo Mineiro, Uberaba/MG - Brasil
}

\begin{abstract}
Resumo
Objetivou-se avaliar os padrões alimentares, de atividade física e o grau de insatisfação com a imagem corporal em mulheres frequentadoras de academia de atividade física. 30 mulheres participaram do estudo. Os instrumentos utilizados foram: Teste de Atitudes Alimentares (EAT-26), Teste de Investigação Bulímica de Edimburgo (BITE), Questionário de Imagem Corporal (BSQ), Inventário de Sentimentos sobre Atividade Física (ISAF) e Índice de Massa Corporal (IMC). Quanto aos resultados, 40\% das participantes apresentavam alteração no IMC, 9,9\% apresentaram comportamento de risco para o desenvolvimento de transtorno alimentar, 57,7\% apresentaram alguma distorção na imagem corporal e 30\% mostraram atitude positiva com relação à prática de atividade física. Mulheres na faixa de 26 a 33 anos apresentaram mais atitudes alimentares patológicas do que as demais. Sugere-se a realização de estudos complementares com foco nas práticas de promoção da saúde envolvendo frequentadoras de academia de atividade física.

Palavras-chave: Anorexia nervosa; Bulimia; Atividade física.
\end{abstract}

Eating patterns and body image in women attending fitness centers

\begin{abstract}
This study aimed to evaluate dietary patterns, physical activity and body image dissatisfaction degree among women who practice physical activity. 30 women participated in the study. The instruments used were: Eating Attitudes Test (EAT26), Bulimic Investigatory Edimburgo Test (BITE), Body Image Questionnaire (BSQ), Inventory of Feelings about Physical Activity (ISAF, in portuguese) and the Body Mass Index. In terms of results, $40 \%$ of participants had negative results in BMI measurement, 9,9\% showed risk behavior to the development of eating disorders, $57,7 \%$ showed body image distortion and 30\% showed a very positive attitude towards physical activity. Women aged 26 to 33 years had more pathological eating attitudes than the others. Further studies with focus on health promotion practices considering women who does physical activity and has eating disorders high-risk behavior is suggested. Keywords: Nervous anorexia; Bulimia; Physical activity.
\end{abstract}

Patrones alimentarios e imagen corporal en mujeres frecuentadoras de gimnasio

\section{Resumen}

Este estudio tuvo como objetivo evaluar los hábitos alimentarios, la actividad física y el grado de insatisfacción en lo que se refiere a la imagen corporal de mujeres que van al gimnasio. Treinta mujeres participaron de este estudio. Los instrumentos utilizados fueron el Test de Actitudes Alimentarias (EAT-26), Prueba Investigadora de Edimburgo (BITE), el Cuestionario de Imagen Corporal (BSQ), Inventario de los Sentimientos sobre la Actividad Física (ISAF) y el Índice de Masa Corporal (IMC). El 40\% de las participantes mostraron cambios en el IMC, el 9,9\% tenían comportamiento de riesgo para el desarrollo de trastornos alimentarios, el 57,7\% mostraron una cierta distorsión en la imagen corporal y el $30 \%$ mostraron una actitud positiva en la práctica de actividades física. Las mujeres de 26 a 33 años presentaron mayor actitud patológica que las otras. Se sugiere realizar más estudios con enfoque en las prácticas de promoción de la salud con la participación de mujeres que frecuentan el gimnasio.

Palabras clave: Anorexia nerviosa; Bulimia; Actividad física.

A constante referência aos quadros psicopatológicos que compõem os transtornos alimentares (TA), especificamente a anorexia nervosa (AN) e bulimia nervosa (BN), tem alertado os profissionais da área da saúde e despertado o interesse tanto pela compreensão dessas patologias, em termos de diagnóstico e tratamento, quanto de prevenção e promoção da saúde, o que se situa no âmbito das propostas das políticas públicas (Fitzpatrick, Moye, \& Hostee, 2010; Rieger, Van Buren \& Bishop, 2010; Souza, 2011).

A incidência da AN é de, pelo menos, 8/100 mil indivíduos da população por ano (Hoek \& van Hoeken, 2003). Em relação à $\mathrm{BN}$, a taxa de incidência é de 30/100 mil indivíduos da população. As estimativas de prevalência de TA ao longo da vida são de $0,9 \%$ para $\mathrm{AN}$ e $1,5 \%$ para $\mathrm{BN}$, no caso das mulheres (Hudson, Hiripi, Pope Jr. \& Kessler, 2007).

As causas dos TA são multifatoriais, envolvendo fatores biológicos, psicológicos e socioculturais, como a busca por um corpo magro e esguio, a pressão por ser magro e também a procura por cirurgias plásticas (Fairburn, 2008; Mitchell, Agras \& Wonderlich, 2007; Tanofsky-Kraff, Wilfley \& Young, 2010). A literatura vem mostrando que os TA manifestam-se na adolescência e idade adulta, quando mudanças no corpo afetam as mulheres em geral e nitidamente as atletas femininas, quanto à aparência e performance (Oliveira, Bosi, Vigário \& Vieira, 2003; Pinto, Camargo, Novo \& Hübner, 2009; Sicchieri, 2005).

Fatores como baixa autoestima e distorção da imagem corporal reforçam a busca de um emagrecimento incessante, levando à prática de 
exercícios físicos em excesso, jejum e uso de laxantes ou diuréticos de uma forma ainda mais intensa (Alves, Calvo, Neves \& Vasconcelos, 2008; Cordás, 2004; Scorsolini-Comin, Souza \& Santos, 2010). A imagem corporal é um conceito que envolve três componentes: o perceptivo, relacionado com a percepção do corpo, e a uma estimativa do tamanho corporal e do peso; o subjetivo, referente à satisfação com a aparência, juntamente com a ansiedade e a preocupação relacionados a ela; e o comportamental, que consiste em situações evitadas em virtude do desconforto causado pela aparência corporal (Cordás, 2004). Adolescentes com esses comportamentos apresentam maior risco para o desenvolvimento de TA quando comparadas as adolescentes satisfeitas com sua imagem corporal (Alves \& cols., 2008).

Estudos têm comprovado que o público de frequentadores de academia tem características próprias que os diferenciam de outros grupos (atletas, indivíduos com TA, população em geral) (Hirschbruch, 2003; Hirschbruch \& Carvalho, 2002). É necessária a criação de protocolos específicos para conhecer melhor as peculiaridades desse grupo e de seus subgrupos, para embasar a atuação dos profissionais que trabalham nessa área (Damasceno \& cols., 2011; Hirschbruch, Fisberg \& Mochizuki, 2008; Meyer, Blissett, Alberry \& Sykes, 2013; Taranis \& Meyer, 2010; Villas Bôas, Ferreira, Souza \& Coelho, 2010).

Como a população de praticantes de atividades físicas é considerada importante grupo de risco para o aparecimento desses transtornos (Beals \& Manore, 2002), este estudo objetivou avaliar os padrões alimentares, de atividade física e grau de insatisfação com a imagem corporal em mulheres de 12 a 39 anos frequentadoras de academia de atividade física.

\section{Método}

\section{Tipo de estudo}

Trata-se de um estudo de caráter descritivo, correlacional e de corte transversal, desenvolvido em uma abordagem quantitativa.

\section{Local e contexto do estudo}

$O$ estudo foi realizado em uma academia particular de atividade física exclusivamente feminina, localizada em região urbana e central de uma cidade de médio porte do interior do estado de São Paulo. Podem se matricular nessa academia mulheres de qualquer idade e que não apresentem impedimentos físicos para prática de exercícios. À época da coleta dos dados, estavam matriculadas 120 mulheres, com idade mínima de quatro anos e máxima de 68 anos. A maior parte das mulheres matriculadas era de classe média e alta, pelo critério ABIPEME (2008).

\section{Participantes}

Participaram do estudo 30 mulheres praticantes de atividade física de uma academia durante o ano de 2009. Entre os critérios de inclusão, destacamos: (a) ser do sexo feminino e ter idade entre 12 e 39 anos. A razão para tal critério baseou-se na vasta revisão de literatura realizada, que aponta ser este o perfil de risco para a ocorrência de TA; (b) estar matriculada na academia onde o estudo foi realizado; (c) não possuir indícios aparentes de comprometimento cognitivo, físico e psicológico. Nenhuma mulher se negou a participar do estudo.

\section{Instrumentos}

(a) Teste de Atitudes Alimentares (EAT-26) (Garner \& Garfinkel, 1979), traduzido para o português (Nunes \& cols., 1994) e validado (Bighetti, 2003). Revela a gravidade de preocupações típicas de pacientes com TA, em especial a AN, como, por exemplo, a intenção de emagrecer e o medo de engordar, as práticas de dietas, comportamentos purgativos e controle excessivo da ingestão alimentar. É um questionário de autorrelato composto por 26 itens e apresenta seis opções de resposta. Possui três fatores: dieta (13 itens), $\mathrm{BN}$ (6 itens) e controle oral (7 itens). Aplicado o instrumento, os escores obtidos em cada questão do EAT-26 foram somados e computados para cada pessoa avaliada. Caso o total de escores encontrado fosse igual ou maior que 21, o EAT-26 seria considerado positivo (EAT-26+) e confirmada a presença de atitudes alimentares patológicas e risco para o desenvolvimento de TA. Os autores que desenvolveram o EAT-26 utilizaram um ponto de corte (cut-off) de 20 escores. No presente estudo foi adotado o cut-off de 21 pontos, pois este apresenta sensibilidade e especificidade altamente satisfatórias e por estar sendo adotado por outros autores nacionais (Assunção, Cordás \& Araújo, 2002; Bighetti, 2003; Nunes, 1997), o que permite comparações entre os resultados.

(b) Teste de Investigação Bulímica de Edimburgo - BITE (Henderson \& Freeman, 1987). Avalia predominantemente comportamentos bulímicos, como a ingestão excessiva de alimentos (binge eating), a provocação de vômitos, a realização de jejum e dieta e o uso de anorexígenos, de laxantes e de diuréticos. É um questionário autoaplicativo composto de 33 questões, com duas subescalas: de sintomas e de gravidade. A escala de sintomas classifica-os em 3 grupos de escores: altos (20 pontos ou mais), médios (10 a 19 pontos) e baixos (abaixo de 10 pontos), correspondendo, respectivamente, a comportamentos alimentares anormais, padrões alimentares não usuais e ausência de anormalidade no comportamento alimentar (Oliveira \& cols., 2003). A escala de gravidade é constituída pelos itens 6,7 e 27 do instrumento e mede a gravidade do comportamento compulsivo pela frequência de atitudes, sendo analisada para os casos 
em que a pontuação na escala de sintomas é superior a 10, ramificando-se em três grupos: alto (10 pontos ou mais), moderado (5 a 9 pontos) e baixo (0 a 4 pontos). O instrumento foi traduzido para o português (Cordás \& Hochgraf, 1993), e evidências de validade para o contexto brasileiro podem ser encontradas em Ximenes e cols.(2011).

(c) Questionário de Imagem Corporal (BSQ Body Shape Questionnaire) (Cooper, Taylor, Cooper \& Fairburn, 1987). Mede as preocupações com a forma do corpo, autodepreciação e a sensação de estar "gorda". É um instrumento autoaplicativo com 34 perguntas com respostas do tipo likert de seis pontos (nunca, raramente, às vezes, frequentemente, muito frequentemente, sempre). A classificação dos resultados foi feita pelo total de pontos obtidos e reflete os níveis de preocupação com a imagem corporal. Obtendo resultado menor ou igual a 80 pontos, é constatado um padrão de normalidade e tido como ausência de distorção da imagem corporal. Resultado entre 81 e 110 pontos é classificado como leve distorção da imagem corporal; entre 111 e 140 é classificado como moderada distorção da imagem corporal; e acima de 140 pontos, a classificação é de presença de grave distorção da imagem corporal (Cordás \& Castilho, 1994). Estudos de validade para o contexto brasileiro foram realizados por Di Pietro (2002).

(d) Inventário de Sentimento sobre Atividade Física (ISAF) (Nielsen \& Corbin, 1986, citado por Santos, 2005). Trata-se de um instrumento autoaplicativo com 12 questões que avaliam a disposição e variação do estado afetivo em relação à prática de atividade física. Apresenta cinco opções de resposta (discordo plenamente, discordo, incerto, concordo e concordo plenamente). Os itens 1, 5, 6, 7, 9 e 12 são pontuados de 1 a 5; os itens 2, 3, 4, 8, 10 e 11 são pontuados de 5 a 1 (Santos, 2005). A avaliação segue a pontuação no inventário, sendo que resultados entre 54 e 60 pontos indicam estado muito favorável sobre a atividade física, ao passo que de 12 a 17 indicam estados muito desfavoráveis. $\mathrm{O}$ instrumento ainda não foi validado para o contexto brasileiro, mas aplicado em outros estudos (Santos, 2005).

(e) Índice de Massa Corpórea (IMC). A classificação do IMC por idade foi o recomendado pela Organização Mundial da Saúde (WHO, 1995). Este trabalho seguiu a classificação do peso corporal proposta por Poirier e cols. (2009). Durante a medição, as mulheres estavam descalças e usando roupas leves. A partir das medidas obtidas, foram calculados os parâmetros segundo orientações de Jackson, Pollock e Warda (1980). Os inventários utilizados são de autoaplicação, podendo esta ser individual ou coletiva.

\section{Procedimento}

Inicialmente, os pesquisadores estabeleceram o rapport com as participantes, fornecendo as informações e os esclarecimentos necessários acerca dos objetivos e procedimentos concernentes à pesquisa. As participantes assinaram o Termo de Consentimento Livre e Esclarecido e, posteriormente, preencheram os instrumentos autoaplicáveis. Em seguida, passaram pela avaliação do IMC com a colaboração da educadora física contratada pela academia. Um teste piloto foi realizado com cinco voluntárias para verificar o tempo necessário para a aplicação dos testes. Assim, a coleta dos dados seguiu uma sequência mais adequada, evitando a fadiga dos participantes. A literatura na área costuma apresentar a sequência de aplicação dos testes aqui utilizados iniciando pelo EAT, BITE, BSQ (Bosi \& cols., 2008), sendo que, no caso, prosseguimos com a aplicação do ISAF, finalizando com as medidas de dobras cutâneas, não utilizadas na presente análise. Após a coleta, as respostas das participantes foram organizadas em um banco de dados que continha todas as informações necessárias, bem como os escores dos instrumentos categorizados por cada participante por meio da atribuição de um número de protocolo. Este estudo foi aprovado pelo Comitê de Ética em Pesquisa da instituição de origem dos pesquisadores (Processo 00122/2008).

\section{Análise dos dados}

Os dados obtidos com a aplicação dos instrumentos foram transpostos para o Software SPSS for Windows versão 17.0 e categorizados, a partir de números de identificação, por participante. A descrição geral dos dados foi apresentada por meio de frequências simples e relativas, bem como por meio das médias e desvios padrão. As participantes foram divididas em três grupos de acordo com o critério etário para verificação de possíveis influências da idade sobre seu comportamento alimentar, conforme orientações da literatura (Pinto \& cols., 2009; Sicchieri, 2005). Em seguida, foi feito o teste de normalidade de Kolmogorov-Smirnov, para determinar o tipo de prova estatística aplicada. Visando compreender melhor a atitude alimentar das participantes, foram feitas provas correlacionais (correlação de Pearson) e provas de análise de diferenças de grupos por faixa etária (Oneway ANOVA), considerando nível de significância de $5 \%$.

\section{Resultados}

\section{Caracterização da amostra e do comportamento alimentar}

Os inventários foram aplicados em 30 alunas que estavam iniciando a prática de exercícios físicos em uma academia exclusivamente feminina. Foram compostos três grupos: (a) Grupo A, de 18 a 25 anos de idade ( $n=14)$; (b) Grupo B, de 26 a 33 anos $(n=8)$; e 
(c) Grupo C, de mulheres acima de 33 anos $(n=8)$. A amostra total $(n=30)$ apresentou idade média de 27,07 anos $(\mathrm{DP} \pm 8,02)$, sendo a idade mínima 18 e a máxima 39 anos. Os resultados sobre índice de massa corporal (IMC) e o perfil alimentar das participantes encontramse expostos na Tabela 1, que apresenta os resultados descritivos sobre o índice de massa corporal, os comportamentos alimentares e a atitude sobre atividades físicas, em termos de frequência total e relativa $(n=30)$.

Tabela 1. Resultados descritivos sobre o índice de massa corporal, os comportamentos alimentares e a atitude sobre atividades físicas

\begin{tabular}{|c|c|c|c|c|c|c|c|c|c|c|c|}
\hline \multicolumn{2}{|c|}{ IMC } & \multicolumn{2}{|c|}{ EAT-26 } & \multicolumn{2}{|c|}{ BSQ } & \multicolumn{2}{|c|}{$\begin{array}{c}\text { BITE } \\
\text { Sintomas }\end{array}$} & \multicolumn{2}{|c|}{$\begin{array}{c}\text { BITE } \\
\text { Gravidade }\end{array}$} & \multicolumn{2}{|c|}{ SAF } \\
\hline & $n(\%)$ & & $n(\%)$ & & $n(\%)$ & & $n(\%)$ & & $n(\%)$ & & $n(\%)$ \\
\hline $\begin{array}{l}\text { Baixo Peso/ } \\
\text { Desnutrição }\end{array}$ & $\begin{array}{c}01 \\
(3,3)\end{array}$ & Positivo & $\begin{array}{c}03 \\
(9,9)\end{array}$ & Normal & $\begin{array}{c}13 \\
(43,3)\end{array}$ & Baixo & $\begin{array}{c}29 \\
(96,7)\end{array}$ & Baixo & $\begin{array}{c}04 \\
(16,7)\end{array}$ & $\begin{array}{l}\text { Muito } \\
\text { favorável }\end{array}$ & $\begin{array}{c}09 \\
(30,0)\end{array}$ \\
\hline Normal & $\begin{array}{c}18 \\
(60,0)\end{array}$ & Negativo & $\begin{array}{c}27 \\
(90,1)\end{array}$ & Leve & $\begin{array}{c}12 \\
(37,7)\end{array}$ & Moderado & $\begin{array}{c}01 \\
(3,3)\end{array}$ & Moderado & $\begin{array}{c}11 \\
(12,3)\end{array}$ & Favoráveis & $\begin{array}{c}16 \\
(53,0)\end{array}$ \\
\hline Sobrepeso & $\begin{array}{c}05 \\
(16,7)\end{array}$ & & & Moderada & $\begin{array}{c}04 \\
(16,7)\end{array}$ & Alto & & Alto & $\begin{array}{c}15 \\
(50,0)\end{array}$ & Neutro & $\begin{array}{c}05 \\
(17,0)\end{array}$ \\
\hline Obesidade & $\begin{array}{c}06 \\
(20,0)\end{array}$ & & & Grave & $\begin{array}{c}01 \\
(3,3)\end{array}$ & & & & & $\begin{array}{c}\text { Muito } \\
\text { desfavorável }\end{array}$ & \\
\hline
\end{tabular}

IMC=Índice de massa corporal; EAT-26=Teste de Atitudes Alimentares; BSQ=Questionário de Imagem Corporal; BITE=Teste de Investigação Bulímica de Edimburgo; ISAF=Inventário de Sentimentos sobre Atividade Física

O IMC médio entre todas as mulheres foi de $26,49 \mathrm{~kg} / \mathrm{m}^{2}( \pm 7,21)$, ficando o IMC mínimo em 18,40 e máximo em 53,40. No sexo feminino, valores entre 19 a $24 \mathrm{~kg} / \mathrm{m}^{2}$ são considerados normais; menores que $19 \mathrm{~kg} / \mathrm{m}^{2}$ representam baixo peso/desnutrição; acima de $25 \mathrm{~kg} / \mathrm{m}^{2}$, tem-se sobrepeso; e valores maiores que $30 \mathrm{~kg} / \mathrm{m}^{2}$ determinam obesidade. Como pode ser observado por meio da Tabela 1, uma parcela relevante da amostra do presente trabalho (20\%) apresentava obesidade, enquanto 3,3\% encontrava-se com baixo peso/desnutrição. Esse índice (IMC) é um indicativo da proporção do peso corporal em virtude da altura do indivíduo.

Com relação às atitudes alimentares e comportamentos alimentares, foram feitas as análises dos inventários EAT-26 e BITE. A análise dos resultados do teste EAT-26 mostrou que 9,9\% $(n=3)$ apresentaram EAT-26+, indicando a presença de atitudes alimentares patológicas e risco para o desenvolvimento de TA. Com relação aos comportamentos alimentares, os resultados do BITE indicaram que apenas $3,3 \%$ da amostra $(n=1)$ apresentavam comportamentos alimentares não usuais.

Com relação à imagem corporal, os resultados do BSQ mostraram que $37,7 \%(n=12)$ apresentaram leve distorção da imagem corporal, 16,7\% $(n=4)$ apresentaram distorção moderada da imagem corporal e 3,3\% $(n=1)$ apresentaram grave distorção da imagem corporal. Os resultados mostraram, ainda, que $30 \%$ da amostra $(n=9)$ tinha um sentimento muito favorável sobre atividades físicas e que nenhuma integrante da amostra era muito desfavorável às atividades físicas.

Após caracterização das participantes e dos comportamentos alimentares e de imagem corporal, os dados foram submetidos à prova de normalidade de Kolmogorov-Smirnov e demonstraram apresentar distribuição normal (K-S entre 0,06 e 0,98), permitindo o uso de provas paramétricas para a análise das correlações e das diferenças entre subgrupos.

\section{Correlações entre variáveis}

Os resultados descritos na Tabela 2 mostraram que existe uma moderada correlação positiva $(r=0,55$; $p \leq 0,001)$ entre o IMC e os resultados do BSQ. Essa correlação indica que as pessoas com maior índice de massa corporal tendem a apresentar maior distorção de sua imagem corporal. Os resultados da BSQ também mostraram moderada correlação positiva com os resultados da EAT $(r=0,49 ; p \leq 0,001)$, indicando a distorção da imagem corporal associada ao desenvolvimento de atitudes alimentares perigosas para o desenvolvimento de TA.

\section{Diferenças entre grupos}

A literatura indica que os TA afetam de modo mais significativo as mulheres no início da adolescência (Pinto \& cols., 2009; Sicchieri, 2005), aproximadamente aos 12 anos de idade. Visando verificar a possível interferência da idade sobre o perfil alimentar, as participantes foram subdivididas em três faixas etárias e seus resultados foram comparados. Embora a amostra seja composta por mulheres com, no mínimo, 18 anos e, portanto, não mais na fase da adolescência, a hipótese era a de que pudesse haver diferenças de acordo com a idade. A Tabela 3 apresenta as análises sobre as diferenças de grupos por faixa etária para o comportamento alimentar. 
Tabela 2. Correlações entre comportamentos alimentares e a atitude sobre atividades físicas

\begin{tabular}{lccccccc}
\hline & Idade & IMC & SAF & BSQ & EAT & $\begin{array}{c}\text { BITE - } \\
\text { sintomas }\end{array}$ & $\begin{array}{c}\text { BITE - } \\
\text { gravidade }\end{array}$ \\
\hline Idade & 1 & $-0,18$ & 0,26 & $-0,23$ & 0,01 & $-0,15$ & $-0,34$ \\
IMC & & 1 & $-0,29$ & $0,50^{* *}$ & 0,08 & 0,09 & 0,12 \\
SAF & & 1 & $-0,19$ & 0,02 & $-0,24$ & $-0,05$ \\
BSQ & & & 1 & $0,49 * *$ & 0,11 & $0,51^{* *}$ \\
EAT & & & & 1 & 0,19 & $0,45^{*}$ \\
BITE - sintomas & & & & & & 1 & 0,27
\end{tabular}

IMC=Índice de massa corporal; EAT-26=Teste de Atitudes Alimentares; BSQ=Questionário de Imagem Corporal; BITE=Teste de Investigação Bulímica de Edimburgo; ISAF=Inventário de Sentimentos sobre Atividade Física

${ }^{* *} \mathrm{p} \leq 0,001 ;{ }^{*} \mathrm{p} \leq 0,05$

Tabela 3. Diferenças de grupos entre os comportamentos alimentares e a atitude sobre atividades físicas

\begin{tabular}{|c|c|c|c|}
\hline & Faixas etárias & Média (DP) & $\mathrm{F}$ \\
\hline \multirow{3}{*}{ IMC } & Idade 18 - 25 & 27,36 & \multirow{3}{*}{0,34} \\
\hline & Idade 26 - 33 & 26,78 & \\
\hline & Acima de 33 & 24,68 & \\
\hline \multirow{3}{*}{ SAF } & Idade $18-25$ & 46,93 & \multirow{3}{*}{0,95} \\
\hline & Idade 26 - 33 & 48,00 & \\
\hline & Acima de 33 & 51,25 & \\
\hline \multirow{3}{*}{ BSQ } & Idade 18 - 25 & 91,64 & \multirow{3}{*}{1,78} \\
\hline & Idade 26 - 33 & 101,38 & \\
\hline & Acima de 33 & 74,00 & \\
\hline \multirow{3}{*}{ EAT } & Idade 18 - 25 & 8,64 & \multirow{3}{*}{$4,11 *$} \\
\hline & Idade 26 - 33 & 16,88 & \\
\hline & Acima de 33 & 6,75 & \\
\hline \multirow{3}{*}{ BITE - sintomas } & Idade 18 - 25 & 3,00 & \multirow{3}{*}{2,70} \\
\hline & Idade 26 - 33 & 4,75 & \\
\hline & Acima de 33 & 1,50 & \\
\hline \multirow{3}{*}{ BITE - gravidade } & Idade $18-25$ & 10,00 & \multirow{3}{*}{1,72} \\
\hline & Idade 26 - 33 & 9,75 & \\
\hline & Acima de 33 & 6,75 & \\
\hline
\end{tabular}

IMC=Índice de massa corporal; EAT-26=Teste de Atitudes Alimentares; BSQ=Questionário de Imagem Corporal; BITE=Teste de Investigação Bulímica de Edimburgo; ISAF=Inventário de Sentimentos sobre Atividade Física

${ }^{* *} \mathrm{p} \leq 0,001 ; * \mathrm{p} \leq 0,05$

As análises mostraram que as mulheres entre 26 a 33 anos foram as que apresentaram escores mais elevados na escala de atitudes alimentares patológicas $(F=4,11 ; \quad p \leq 0,05)$. Não houve outras diferenças estatisticamente significantes sobre os comportamentos alimentares ou nas atitudes sobre atividades físicas por faixa etária, rejeitando-se a hipótese de influência da idade nesses comportamentos.

\section{Discussão}

Os principais motivos pelos quais as pessoas iniciam programas de atividade física são a insatisfação com o próprio corpo ou mesmo pela imagem que se tem dele (Assunção, Cordás, \& Araújo, 2002). Tal consideração pode ser utilizada para explicar que mais da metade das mulheres que participaram do estudo $(54 \%)$ e que possuíam IMC normal (eutrófico) gostariam de pesar menos ou sentiam-se desconfortáveis em relação ao peso, gordas, até mesmo muito gordas e $50 \%$ das mulheres apresentaram comportamento alimentar não usual. Esses achados Psico-USF, Bragança Paulista, v. 18, n. 3, p. 457-470, set/ dez 2013 podem indicar que, independentemente do peso que as participantes acreditem ter, o medo de engordar está sempre presente para a maioria delas, o que pode repercutir nas distorções da imagem corporal encontradas de modo significativo na amostra.

Nesse sentido, a academia pode e deve implementar formas de acompanhar o desempenho de seus alunos não apenas em termos de rendimento, mas de possíveis práticas ou comportamentos que indiquem a instalação de sintomas de TA com o tempo. Como $57,7 \%$ das participantes apresentaram alguma distorção da imagem corporal, problematiza-se que isso ocorra em resposta a uma má aceitação das mudanças corporais, principalmente do peso, associado a fatores psicológicos individuais, familiares e socioculturais, podendo predispor a um TA. Considerações similares foram apontadas por Garfinkel e Garner (1979) e por Lock (2011). Desse modo, os possíveis efeitos da prática regular de exercícios físicos em academia sobre a imagem corporal podem ser estudados como forma de se desenvolverem recursos para o bem-estar físico e emocional dessas mulheres. 
O EAT revela a gravidade de preocupações típicas de pacientes com os TA, em especial a AN, como, por exemplo, a intenção de emagrecer e o medo de engordar, as práticas de dietas, comportamentos purgativos e controle excessivo da ingestão alimentar (Oliveira \& cols., 2003). A incidência de risco para TA observada no presente estudo encontra-se abaixo dos índices apresentados nos estudos nacionais que utilizaram o mesmo instrumento. Embora não tenha sido realizada uma avaliação dos sintomas de TA com uma intenção diagnóstica, esses dados devem ser analisados em relação aos possíveis percursos dessas frequentadoras ao longo do tempo, na academia. Nesse sentido, há que se destacar que a prática de atividade física pode ser tanto instaladora de mecanismos protetivos quanto ao aparecimento dos TA, como também ser favorecedora do desenvolvimento desses transtornos, esta última consideração amplamente corroborada pela literatura científica (Hirschbruch \& Carvalho, 2002; Hirschbruch, Fisberg \& Mochizuki, 2008). As atividades físicas devem, desse modo, ser compreendias para além da redução de peso, evocando a reflexão de profissionais de saúde em torno dessa problemática.

No presente estudo, a maior taxa de indivíduos com sintomas para o desenvolvimento de TA foi observada no grupo das mulheres entre 26 a 33 anos, o que é contrário à literatura científica na área, que atesta que esses transtornos atingem mais as adolescentes (Alves \& cols., 2008; Oliveira \& cols., 2003; Pinto \& cols., 2009; Sicchieri, 2005). Não houve indicações estatísticas suficientes para explicar tal achado, o que destaca a necessidade de mais estudos que investiguem os sintomas de TA em frequentadoras de academia. $\mathrm{Na}$ contemporaneidade, todas as faixas etárias estão mais expostas aos padrões rígidos de beleza, o que pode contribuir para o aumento do número de casos em diferentes populações, inclusive na de homens (Melin \& Araújo, 2002) e de crianças. Tal consideração aponta para o desenvolvimento de estratégias de prevenção e promoção de saúde que atendam a diferentes idades e que compreendam as academias de exercícios como espaços privilegiados para tais investigações.

Em outros estudos nacionais (Damasceno \& cols., 2011; Pinto \& cols., 2009), o ambiente universitário foi destacado, notadamente o de cursos ligados direta ou indiretamente com saúde, corpo, bem-estar e beleza. No esteio dessas pesquisas com universitárias praticantes de exercício físico, as academias podem figurar como um importante campo de estudo (Meyer \& cols., 2013). Indivíduos afetivamente favoráveis à atividade física tendem a ser mais estáveis com relação à prática de exercícios e esportes e a frequentarem academias. A soma de necessidades (estética, saúde, entre outras) torna a atividade física mais significativa, sendo confirmado este fato pelo inventário (SAF). Com relação ao sentimento sobre a atividade física, verifica-se que a pontuação dos escores foram favorável e muito favorável, totalizando $83 \% \quad(n=25)$, o que revela um aumento da importância para a sua manutenção. Além disso, 30\% mostraram atitude positiva com relação à prática de atividade física, o que pode favorecer o desenvolvimento de programas de promoção da saúde em academias, corroborando a necessidade de estudos nesse contexto (Koyuncu \& cols., 2010).

A atividade física está associada a uma série de benefícios para a saúde física. É conhecido também seu efeito positivo na saúde mental, sendo utilizada com sucesso como tratamento coadjuvante em quadros de depressão, ansiedade e outros fatores estressantes psicossociais (Cordás, 2004). Assim, exigências socioculturais podem determinar o início de uma restrição alimentar e a prática de exercício físico. Podese problematizar, desse modo, que o início da prática de atividade física pode se dar após preocupações (insatisfação) com a imagem corporal. A visão distorcida que as participantes têm do seu próprio corpo pode precipitar o desejo de perder peso, o que pode estar relacionado à aquisição de hábitos alimentares considerados disfuncionais e também ao início das atividades em academia. As mulheres com atitudes e comportamentos alimentares considerados disfuncionais estão mais propensas a acreditar que a prática de exercício físico pode prevenir consequências sociais negativas e a se engajarem nessas atividades (Meyer et al., 2013). Desse modo, a população feminina frequentadora de academia compõe um público-alvo que deve ser investigado com maior atenção e profundidade.

\section{Considerações finais}

Como conclusão do estudo, houve presença de sintomas moderados e graves relacionados aos comportamentos alimentares em $62,3 \%$ das participantes, e 57,7\% apresentaram alguma distorção da imagem corporal que pode estar associada a atitudes alimentares perigosas para o desenvolvimento de TA. A imagem corporal distorcida também manteve relação com o agravamento dos sintomas comportamentais alimentares. Os escores mais elevados sobre as atitudes alimentares patológicas não foram encontrados nas participantes mais jovens, em oposição à literatura na área.

Uma das limitações do estudo refere-se ao fato de se tratar de uma amostra reduzida e de conveniência coletada em apenas uma academia. Desse modo, seus achados e suas conclusões devem ser apreciados com parcimônia. A dificuldade de encontrar estudos nacionais desenvolvidos em academias para possíveis comparações também deve ser assinalada. Não foi possível estabelecer uma relação causal entre os sinais e sintomas de TA em mulheres e o ambiente de academia de exercício, haja vista que a amostra foi composta apenas por mulheres que estavam iniciando a 
prática de exercícios em academia. É desejável a realização de estudos longitudinais nesses ambientes acompanhando as participantes ao longo do tempo, podendo-se comparar os escores obtidos nos instrumentos em diferentes momentos da prática de exercício e verificar seus efeitos em relação às atitudes e comportamentos alimentares. Esses estudos podem contribuir para que se compreenda de que modo a prática do exercício em academia, a longo prazo, pode ser favorecedora ou não de posicionamentos mais positivos acerca do bem-estar relacionado à atividade física.

A partir dessas considerações, destaca-se a atuação de profissionais envolvidos no tratamento dos TA para além de hospitais e centros de saúde, compreendendo que as academias de exercício físico podem ser ambientes fortuitos não apenas para uma rotina de vida considerada mais saudável, mas também para a promoção da saúde. Assim, o reconhecimento da presença de indicadores dos TA nessa população poderá colaborar na compreensão de seu aparecimento e desenvolvimento, produzindo valioso conhecimento para a proposta de práticas preventivas nessa população e estratégias de atendimento mais condizentes com suas necessidades. Formar profissionais para atuação nesse contexto de modo interdisciplinar pode ser um mote de discussão nos currículos dos cursos de graduação e pós-graduação das áreas envolvidas, entre elas a Psicologia.

\section{Referências}

Alves, E., Calvo, M. C. M., Neves, J. \& Vasconcelos, F. A. G. (2008). Prevalência de sintomas de anorexia nervosa e insatisfação com a imagem corporal em adolescentes do sexo feminino do Município de Florianópolis, Santa Catarina, Brasil. Cadernos de Saúde Pública, 24(3), 503-512.

Associação Brasileira dos Institutos de Pesquisa de Mercado (ABIPEME) (2008). Critério de classificação socioeconômico do Brasil (CCSEB). São Paulo: ABA, ANEP, ABIPEME.

Assunção, S. S. M., Cordás, T. A. \& Araújo, L. A. S. B. (2002). Atividade física e transtornos alimentares. Revista Psiquiatria Clínica, 29(1), 4-13.

Beals, K. A., \& Manore, M. M. (2002). Disorders of the female athlete triad among collegiate athletes. International Journal of Sport Nutrition and Exercise Metabolism, 12(3), 281-293.

Bighetti, F. (2003). Tradução e validação do Eating Attittudes Test (EAT 26) em adolescentes do sexo feminino na cidade de Ribeirão Preto-SP. Dissertação de Mestrado, Escola de Enfermagem de Ribeirão Preto, Universidade de São Paulo, Ribeirão Preto, SP.
Bosi, M. L. G., Luiz, R. R., Uchimura, K. Y., \& Oliveira, F. P. (2008). Comportamento alimentar e imagem corporal entre estudantes de educação física. Jornal Brasileiro de Psiquiatria, 57(1), 28-33.

Cooper, P. J., Taylor, M., Cooper, Z. \& Fairburn, C. G. (1987). The development and validation of the Body Shape Questionnaire. International Journal of Eating Disorders, 6, 485-494.

Cordás, T. A. (2004). Transtornos alimentares: classificação e diagnóstico. Revista de Psiquiatria Clinica, 31(4), 154-157.

Cordás, T. A., \& Hochgraf, P. O. (1993). "BITE": instrumento para avaliação da bulimia nervosa. Jornal Brasileiro de Psiquiatria, 42, 141-144.

Cordás, T. A. \& Castilho, S. (1994). Imagem corporal nos transtornos alimentares, instrumento de avaliação: Body Shape Questionnaire. Psiquiatria Biológica, 2, 17-21.

Damasceno, M. L., Schubert, A., Oliveira, A. P., Sonoo, C. N., Vieira, J. L. L. \& Vieira, L. F. (2011). Associação entre comportamento alimentar, imagem corporal e esquemas de gênero do autoconceito de universitárias praticantes de atividades físicas. Revista Brasileira de Atividade Física \& Sańde, 16(2), 138-143.

Di Pietro, M. C. (2002). Validade interna, dimensionalidade e desempenho da escala BSQ - "Body Shape Questionnaire" em uma população de estudantes universitários. Dissertação de Mestrado, Escola Paulista de Medicina da Universidade Federal de São Paulo, São Paulo.

Fairburn, C. G. (2008). Cognitive behavioral therapy and eating disorders. Nova Iorque: Guilford Press.

Fitzpatrick, K., Moye, A. \& Hostee, R. (2010). Adolescent focused therapy for adolescent anorexia nervosa. Journal of Contemporary Psychotherapy, 40, 31-39.

Garner, D. M. \& Garfinkel, P. E. (1979). The eating attitudes test: an index of the symptoms of anorexia nervosa. Psychology Medicine, 9(2), 273-279.

Henderson, M. \& Freeman, C. P. L. (1987). A selfrating scale for bulimia: The BITE. Brazilian Journal of Psychiatry, 50, 18-24.

Hirschbruch, M. D. (2003). Consumo de suplementos por jovens frequentadores de academias de ginástica em São Paulo. Tese de Doutorado, Universidade Federal de São Paulo, São Paulo.

Hirschbruch, M. D. \& Carvalho, J. R. (2002). Nutrição esportiva: uma visão prática. Em M. D. Hirschbruch \& J. R. Carvalho. A nutrição em academias: aspectos práticos (pp. 123-158). São Paulo: Manole. 
Hirschbruch, M. D. Fisberg, M., \& Mochizuki, L. (2008). Consumo de suplementos por jovens frequentadores de academias de ginástica em São Paulo. Revista Brasileira de Medicina do Esporte, 14(6), 539-543.

Hoek, H. W. \& van Hoeken, D. (2003). Review of the prevalence and incidence of eating disorders. International Journal of Eating Disorders, 34, 383-396.

Hudson, J. I., Hiripi, E., Pope Jr., H. G. \& Kessler, R. C. (2007). The prevalence and correlates of eating disorders in the national comorbidity survey replication. Biological Psychiatry, 61(3), 348-358.

Jackson, A. S., Pollock M. L. \& Warda, A. (1980). Generalized equation for predicting body density of women. Medicine Science Sports Exercise, 12, 17582.

Koyuncu, M., Tok, S., Canpolat, M. \& Catikkas, F. (2010). Body image satisfaction and dissatisfaction, social physique anxiety, selfesteem, and body fat ratio in female exercisers and nonexercisers. Social Behavior and Personality, 38(4), 561-570.

Lock, J. (2011). Evaluation of family treatment models for eating disorders. Current Opinion in Psychiatry, 24, 274-279.

Melin, P., \& Araújo, A. M. (2002). Transtornos alimentares em homens: um desafio diagnóstico. Revista Brasileira de Psiquiatria, 24(3), 73-76.

Meyer, C., Blissett, J., Alberry, R. \& Sykes, A. (2013). Beliefs about exercise: relationship to eating psychopathology and core beliefs among young female exercisers. Eating Behaviors, 14, 79-82.

Mitchell, J., Agras, W. S. \& Wonderlich, S. (2007). Treatment of bulimia nervosa: where are we and where are we going? International Journal of Eating Disorders, 40, 95-101.

Nunes, M. A. A. (1997). Prevalência de comportamentos alimentares anormais e práticas inadequadas de controle de peso em mulheres de 12 a 21 anos em Porto Alegre. Dissertação de Mestrado, Universidade Federal de Pelotas, Pelotas, RS.

Nunes, M. A., Bagatini, L. F., Abuchaim, A. L., Kunz, A., Ramos, D., Silva, J. A., Somenzi, L. \& Pinheiro, A. (1994). Distúrbios da conduta alimentar: considerações sobre o teste de atitudes alimentares (EAT). Revista Brasileira de Psiquiatria, 16(1), $7-10$

Oliveira, F. P., Bosi, M. L. M., Vigário, P. S. \& Vieira, R. S. (2003). Eating behavior and body image in athletes. Revista Brasileira de Medicina do Esporte, 9(6), 357-367.
Pinto, A. C. M., Camargo, M. R., Novo, N. F. \& Hübner, C. K. (2009). Transtornos alimentares em alunas da Faculdade de Medicina do Centro de Ciências Médicas e Biológicas da PUC-SP. Revista da Faculdade de Ciências Médicas de Sorocaba, 11(2), 16-20.

Poirier, P., Alpert, M. A., Fleisher, L. A., Thompson, P. D., Sugerman, H. J., Burke, L. E., Marceau, P. \& Franklin, B. A. (2009). Cardiovascular evaluation and management of severely obese patients undergoing surgery: a science advisory from the American Heart Association. Circulation, 120, 8695.

Rieger, E., Van Buren, D., \& Bishop, M. (2010). An eating disorder-specific model of interpersonal therapy (IPT-ED): causal pathways and treatment implications. Clinical Psychology Review, 30, 400-410.

Santos, J. F. S. (2005). Sentimento sobre atividade física em estudantes universitários de Joinville. Revista Brasileira de Atividade Física e Saúde, 10(1), 80-81.

Scorsolini-Comin, F., Souza, L. V. \& Santos, M. A. (2010). Construção de si em grupo de apoio a pessoas com transtornos alimentares. Estudos de Psicologia, 27(4), 467-478.

Sicchieri, J. M. F. (2005). Portadores de transtorno alimentares: da doença a quê? Dissertação de Mestrado, Escola de Enfermagem de Ribeirão Preto, Universidade de São Paulo, Ribeirão Preto, SP.

Siegel, S., \& Castellan, N. J. Jr. (1988). Nonparametric statistics (2a ed). Nova Iorque: McGraw-Hill.

Souza, L. V. (2011). Construindo cuidado: a relação com o profissional de saúde nas práticas discursivas de pessoas diagnosticadas com transtornos alimentares. Tese de Doutorado, Universidade de São Paulo, Ribeirão Preto, SP.

Tanofsky-Kraff, M., Wilfley, D. \& Young, J. (2010). A pilot study of interpersonal psychotherapy for preventing excess weight gain in adolescent girls at-risk for obesity. International Journal of Eating Disorders, 43, 701-706.

Taranis, L. \& Meyer, C. (2010). Perfectionism and compulsive exercise among female exercisers: high personal standards or self-criticism? Personality and Individual Differences, 49, 3-7.

Villas Bôas, M. S., Ferreira, V. A., Souza, C., \& Coelho, R. W. (2010). Nível de satisfação com a imagem corporal e a aparência muscular de praticantes de musculação. Revista da Educação Física (Maringá), 21(3), 493-502.

World Health Organization - WHO (1995). Physical status, the use and interpretation of anthropometry: report of a WHO expert committee. Genebra: Author.

Psico-USF, Bragança Paulista, v. 18, n. 3, p. 445-454, set/ dez 2013 
Ximenes, R. C. C., Colares, V., Bertulino, T., Couto, G. B. L., \& Sougey, E. B. (2011). Versão brasileira do BITE para uso em adolescentes. Arquivos Brasileiros de Psicologia, 63(1), 52-63.

Recebido em 06/09/2011

Reformulado em 13/11/2012 Segunda Reformulação em 29/05/2013 Aprovado em 11/09/2013 
Sobre os autores:

Marcia Cristina Dominguez Fernandes Pinto de Souza é psicóloga pela UNIFAFIBE (Bebedouro/SP), educadora física e especialista em Psicoterapia Comportamental e Cognitiva.

Laura Vilela e Souza é professora adjunta do Departamento de Psicologia da Universidade Federal do Triângulo Mineiro (UFTM) e doutora em Psicologia pela Universidade de São Paulo (USP).

Sabrina Martins Barroso é professora assistente do Departamento de Psicologia da Universidade Federal do Triângulo Mineiro (UFTM) e doutoranda em Saúde Pública pela Universidade Federal de Minas Gerais (UFMG).

Fabio Scorsolini-Comin é professor adjunto do Departamento de Psicologia da Universidade Federal do Triângulo Mineiro (UFTM) e doutor em Psicologia pela Universidade de São Paulo (USP).

Contato com os autores:

Universidade Federal do Triângulo Mineiro (UFTM) - Instituto de Educação, Letras, Artes, Ciências Humanas e Sociais - Departamento de Psicologia

Avenida Getúlio Guaritá, 159, Centro Educacional (Sala 320) - Bairro Abadia - CEP 38025-440

Uberaba/MG - BRASIL

E-mail: lauravilelasouza@gmail.com 